\title{
Annexin A1 regulates EGFR activity and alters EGFR-containing tumour-derived exosomes in head and neck cancers
}

\author{
N. Raulf ${ }^{\text {a }}$, P. Lucarelli ${ }^{\text {b }}$, S. Thavaraj ${ }^{\text { }}$, S. Brown ${ }^{\text {d }}$, J.M. Vicencio ${ }^{\text {e }}$, \\ T. Sauter ${ }^{\text {b }, ~ M . ~ T a v a s s o l i ~}{ }^{\text {a, } *}$
}

a Department of Molecular Oncology, King's College London, Guy's Hospital Campus, Hodgkin Building, London SE1 1UL,

$U K$

${ }^{\mathrm{b}}$ Faculté des Sciences, de La Technologie et de La Communication, University of Luxembourg, 6, Avenue Du Swing,

4367 Belvaux, Luxembourg

${ }^{\mathrm{c}}$ Department of Head and Neck Pathology, Mucosal and Salivary Biology, Guy's Hospital Campus, King's College London, SE1 9RT, UK

d DCT3 Oral and Maxillofacial Histopathology, Department of Head \& Neck Pathology, Guy's Hospital Campus, King's College London, SE1 9RT, UK

${ }^{\mathrm{e}}$ Research Department of Cancer Biology, Cancer Institute, University College London, Paul O'Gorman Building,

72 Huntley Street, London, WCIE 6BT, UK

Received 9 March 2018; received in revised form 29 May 2018; accepted 16 July 2018

\section{KEYWORDS}

Head and neck cancer; EGFR signalling;

Annexin A1

(ANXA1);

Formyl peptide

receptor;

Cetuximab;

Exosomes;

EGFR

phosphorylation;

miR-196a

\begin{abstract}
Background: Head and neck squamous cell carcinoma (HNSCC) is the 6th most common cancer with approximately half a million cases diagnosed each year worldwide. HNSCC has a poor survival rate which has not improved for over 30 years. The molecular pathogenesis of HNSCCs remains largely unresolved; there is high prevalence of p53 mutations and EGFR overexpression; however, the contribution of these molecular changes to disease development and/or progression remains unknown. We have recently identified microRNA miR-196a to be highly overexpressed in HNSCC with poor prognosis. Oncogenic miR-196a directly targets Annexin A1 (ANXA1). Although increased ANXA1 expression levels have been associated with breast cancer development, its role in HNSCC is debatable and its functional contribution to HNSCC development remains unclear.
\end{abstract}

Methods: ANXA1 mRNA and protein expression levels were determined by RNA Seq analysis and immunohistochemistry, respectively. Gain- and loss-of-function studies were performed to analyse the effects of ANXA1 modulation on cell proliferation, mechanism of

\footnotetext{
* Corresponding author: Department of Molecular Oncology, King's College London, Guy's Hospital Campus, Hodgkin Building, London SE1 1UL, UK.

E-mail address: mahvash.tavassoli@kcl.ac.uk (M. Tavassoli).
} 
activation of EGFR signalling as well as on exosome production and exosomal phosphoEGFR.

Results: ANXA1 was found to be downregulated in head and neck cancer tissues, both at mRNA and protein level. Its anti-proliferative effects were mediated through the intracellular form of the protein. Importantly, ANXA1 downregulation resulted in increased phosphorylation and activity of EGFR and its downstream PI3K-AKT signalling. Additionally, ANXA1 modulation affected exosome production and influenced the release of exosomal phosphoEGFR.

Conclusions: ANXA1 acts as a tumour suppressor in HNSCC. It is involved in the regulation of EGFR activity and exosomal phospho-EGFR release and could be an important prognostic biomarker.

(C) 2018 Elsevier Ltd. All rights reserved.

\section{Introduction}

Every year nearly 600,000 new cases of head and neck cancer are diagnosed worldwide, which equals over 1451 cases every day [1]. Incidence rates are rising, and most patients present with advanced stages of the disease which negatively impacts treatment success [1,2]. Additionally, the molecular pathways involved in the development and progression of head and neck squamous cell carcinomas (HNSCC) remain largely unknown. Therefore, there is an important need to identify predictive and prognostic biomarkers and understand their functional contribution to HNSCC development. We recently identified Annexin A1 (ANXA1) as a target of the oncogenic microRNA miR196a [3]. The $37 \mathrm{kDa}$ ANXA1 (also known as lipocortin1) is a member of the annexin superfamily of proteins, which contains multiple calcium and phospholipidbinding proteins implicated in a broad range of cellular functions $[4,5]$. ANXA1 exists in two major forms-a secreted extracellular (cleaved) protein and a full-length intracellular protein [6]. Originally, ANXA1 was identified as a glucocorticoid-regulated anti-inflammatory protein with important functions in the innate and adaptive immune response [7]. These activities were mainly attributed to the externalised form of ANXA1 and are mediated by binding of the protein to formyl peptide receptors (FPRs), 7 transmembrane domain and $G$ protein-coupled receptors [7]. In contrast to the mainly antiinflammatory functions of secreted ANXA1, the fulllength intracellular protein has been associated with a wide range of cellular functions such as cytoskeletal organisation, plasma membrane repair, autophagy and intracellular calcium signalling as well as lipid-mediated signalling pathways [8-11]. Different intracellular localisations and stimulus- or modification-dependent redistributions of the ANXA1 protein have been observed $[12,13]$. A role of ANXA1 in tumour development and progression has been described for different cancer subtypes. While in most cancer types such as breast, cervical, hepatic and pancreatic cancer high levels of ANXA1 have been associated with more aggressive disease, its role in HNSCC remains debatable [14-19]. Interestingly, one of the main risk factors of oral cancer, chewing tobacco, results in the loss of the anti-inflammatory activity of ANXA1 rather than its increase [20].

In recent years, ANXA1 has been linked to epidermal growth factor receptor signalling in cervical cancer [21]. Epidermal growth factor receptor (EGFR), also known as Her1 or ErbB-1, is a receptor tyrosine kinase belonging to the ErbB family of receptors, which also includes ErbB-2, ErbB-3 and ErbB-4 [22]. EGFR is widely considered to be one of the central drivers of oncogenesis in HNSCC [23]. More than $80 \%$ of HNSCC cases exhibit an increased membraneous expression of EGFR, and overexpression of the receptor is linked to radiotherapy-resistance mechanisms [24,25]. Natural ligands of EGFR are growth factors, including EGF and TGF- $\alpha$ [26,27]. Ligand binding results in receptor phosphorylation and activation, although ligandindependent and constitutive EGFR signalling have also been described $[28,29]$. EGFR activation steers among others JAK/STAT signalling as well as the PI3K/ AKT, the RAS/RAF/ERK and the PLC/PKC pathways controlling processes including DNA repair, proliferation, angiogenesis, and inhibition of apoptosis [30]. Several EGFR-targeting drugs have been developed. One of these drugs is cetuximab, an IgG1-chimerised $\alpha$ EGFR monoclonal antibody with high affinity for the extracellular EGF domain of EGFR and thereby preventing ligand binding and receptor activation [31]. However, EGFR targeted drugs so far have shown very limited effect in HNSCC.

Depending on the type of ligand, ligand-activated EGFR can signal from various subcellular compartments unless it is sequestered on intraluminal vesicles (ILVs) in the lumen of multivesicular endosomes where it is delivered to lysosomes for degradation [32]. ANXA1 has been suggested to be important for the formation of the intraluminal vesicles and thus termination of EGFR signalling in cervical cancer [33,34]. 
ILVs are also precursors of a type of extracellular vesicles called exosomes [35]. Interestingly, in addition to its intracellular function, lately, a role of extracellular exosomal EGFR in metastasis formation and tumour immunity has been described [36,37]. In this study, we have investigated the involvement of ANXA1 in HNSCC with the aim of exploiting its potential as a prognostic/predictive biomarker and/or therapeutic target in the cancers of head and neck.

\section{Materials and methods}

\subsection{Cell lines and tissue culture}

The cell lines included in this study were HEK293T cells, the head and neck squamous cell carcinoma (HNSCC) cell lines HN5, HN30, HSC3, H357, UTSCC-34, UTSCC-6A, SCC-22B as well as the breast cancer cell line MCF-7. UTSCC-34 and UTSCC-6A cells were provided by Professor Reidar Grenman, Department of Otorhinolaryngology, Head and Neck Surgery, Turku University and Turku University Hospital, Turku, Finland; SCC-22B cells by Dr. Thomas Carey, University of Michigan, USA; and H357 cells by Dr. Stephen Prime, Department of Oral and Dental Science, University of Bristol, Bristol, UK. HN5 and HSC3 cells were obtained from Professor Barry Gusterson, Department of Pathology, University of Glasgow, UK and Dr. Kazuya Tominaga, Department of Oral Pathology, Osaka Dental University, Japan, respectively. HN30 cells were provided by Dr. Andrew Yeudall, Philips Institute of Oral and Craniofacial Molecular Biology, Virginia Commonwealth University, Richmond, Virginia, USA. HEK293T cells which were used for retrovirus and lentivirus production were a gift from Dr. Lucas Chan, Rayne Institute, King's College London, UK. All cell lines except H357, UTSCC-34, and UTSCC-6A were maintained in Dulbecco's Modified Eagles Medium (DMEM; GE Healthcare, Chalfont St. Giles, UK) supplemented with $10 \%$ foetal bovine serum (FBS), $50-\mu \mathrm{g} / \mathrm{ml}$ streptomycin, $100-\mu \mathrm{g} / \mathrm{ml}$ penicillin and $1 \mathrm{mM}$ sodium pyruvate. UTSCC-34 and UTSCC-6A cells were maintained in DMEM (GE Healthcare, Chalfont St. Giles, UK) supplemented with $10 \%$ FBS, $2 \mathrm{mM}$ L-glutamine, 5-ml nonessential amino acids, 125$\mu \mathrm{g} / \mathrm{ml}$ streptomycin and $250-\mu \mathrm{g} / \mathrm{ml}$ penicillin. For H357 cells, DMEM-F12 supplemented with $10 \%$ FBS, $4 \mathrm{mM}$ L-glutamine, $69 \mathrm{nM}$ hydrocortisone, $50-\mu \mathrm{g} / \mathrm{ml}$ streptomycin, $100 \mu \mathrm{g} / \mathrm{ml}$ penicillin and $1 \mathrm{mM}$ sodium pyruvate was used. The expression of ANXA1 was stably modulated in selected HNSCC cell lines. Lentiviral constructs used for downregulation of ANXA1 have been described previously [3]. Sh-scrambled control and sh-ANXA1 expressing cells were selected using $2 \mu \mathrm{g} / \mathrm{ml}$ puromycin. For treatment with recombinant EGF (Peprotech, London, UK), 500,000 cells per well were seeded in a 6-well plate. The next day, cells were serumstarved and $24 \mathrm{~h}$ later treated with $50-200 \mathrm{ng} / \mathrm{ml} \mathrm{re}-$ combinant EGF (in 5\% trehalose) or 5\% trehalose only for the indicated time points. For cellular proliferation studies with recombinant ANXA1, $100 \mathrm{nM}$ recombinant ANXA1 were included. Recombinant ANXA1 was provided by Prof Mauro Perretti, William Harvey Research Institute, Barts and The London School of Medicine, Queen Mary University of London, UK. pLenti-puro-hANXA1-FL was a kind gift from Dr. Egle Solito, William Harvey Research Institute, Barts and the London School of Medicine and Dentistry, Queen Mary University of London, UK.

\subsection{Western blot analysis}

Immunoblotting was performed as described previously [38]. Briefly, cells were lysed in lysis buffer ( $1 \mathrm{mM} \mathrm{MgCl}$, $12.5 \mathrm{mM}$ HEPES/KOH, pH 7.4, $1 \mathrm{mM}$ EGTA, $1 \%$ Triton-X100) or $10 \times$ RIPA buffer (exosomes) including protease inhibitors. Protein concentration was determined by Bradford assay, and $25-50 \mu \mathrm{g}$ of protein was separated on $8-15 \% 1.5 \mathrm{~mm}$ thick SDS-gels and transferred to nitrocellulose membranes (400 $\mathrm{mA}, 90 \mathrm{~min})$ using the Mini-PROTEAN electrophoresis system combined with the Mini-Trans Blot module (Bio-Rad, Hercules, CA, USA). Afterwards membranes were probed with the antibodies of choice. Antibodies used for immunoblotting were beta-actin, alpha-tubulin (Sigma-Aldrich, St. Louis, MO, USA), EGFR F4, phospho-ERK1/2, phospho-AKT (Ser473) phospho-EGFR (Tyr1068), pan-AKT, phosphoSTAT3 (Tyr705), pan-STAT3, ANXA1 (Cell Signaling, Danvers, MA, USA) and CD63 (MEM-259, GeneTex, Inc., Irvine, CA, USA). Antibodies were used at a concentration of 1:1000 or 1:5000 (beta-actin, alpha-tubulin). Secondary HRP-coupled anti-rabbit (1:2000) and antimouse antibodies (1:1000) were obtained from Fisher Scientific (Loughborough, UK) and Sigma-Aldrich (St. Louis, MO, USA), respectively.

\subsection{Immunofluorescence}

30,000 cells were seeded in duplicate in 8-chamber slides (BD Biosciences, San Jose, CA, USA). The next day, cells were washed twice with $1 \times$ PBS and fixed in $4 \%$ paraformaldehyde for $15 \mathrm{~min}$. Following 2 washing step with $1 \times$ PBS, cells were permeabilised for 15 min using $0.2 \%$ Triton-X100, then washed again and incubated for $30 \mathrm{~min}$ in $3 \%$ bovine serum albumin (BSA) in TBSTween. Cells were incubated at $4{ }^{\circ} \mathrm{C}$ overnight under constant agitation in antibodies of choice (diluted in 3\% BSA in TBS-Tween) or 3\% BSA in TBS-Tween only as negative control. Afterwards, cells were washed twice using $1 \times$ PBS and then incubated with secondary fluorescently tagged antibody for $90 \mathrm{~min}$ at $37^{\circ} \mathrm{C}$ protected from light. Following several washing steps in $1 \times$ PBS, the chambers were removed from the slides, and cells 
were mounted in Vectashield mounting medium containing 4',6-diamidin-2-phenylindole (DAPI; Vector Laboratories; Burlingame, CA, USA). Images were acquired on an Olympus BX61 at $60 \times$ magnification. Antibodies used for immunofluorescence were EGFR F4 and FPR2. The FPR2 antibody was kindly provided by Prof Mauro Perretti, William Harvey Research Institute, Barts and The London School of Medicine, Queen Mary University of London, UK.

\subsection{Lentiviral-based expression modulation}

ANXA1 knockdown and scrambled control cells were generated as described previously [3]. Virus was produced in HEK293T cells. Viral supernatant was harvested after 24, 36, and $48 \mathrm{~h}$, filtered through a $0.45 \mu \mathrm{m}$ filter and immediately used for transduction of target cells. Cells were selected based on their newly acquired antibiotic resistance.

\subsection{Cell proliferation assay}

30,000 cells per well were seeded in triplicate in 6-well plates. Over the following $5 \mathrm{~d}$, cells were trypsinised and counted using a Neubauer chamber. Cell proliferation was also assessed using the 3-(4,5-dimethylthiazol-2-yl)-2,5diphenyltetrazolium bromide (MTT) cell viability assay. Stably modulated cells were seeded in a 96-well plate ( 2000 cells in $150 \mu \mathrm{l}$ medium per well), 6 wells per time point. The next day, the medium was replaced with $150 \mu 1$ $\mathrm{DMEM} \pm 100 \mathrm{nM}$ recombinant ANXA1 or different concentrations of recombinant $\mathrm{EGF}(0.5,5$ and $50 \mathrm{ng} / \mathrm{ml})$ using trehalose as vehicle control. For the following $5 \mathrm{~d}$, $20 \mu \mathrm{l}$ MTT solution (Calbiochem, Watford, UK; $5 \mathrm{mg} / \mathrm{ml}$ MTT in $1 \times$ PBS) was added to each well, and after $2 \mathrm{~h}$ of incubation, $150-\mu 1$ solubilisation solution $(50 \%$ dimethylformamide, $0.2 \%$ glacial acetic acid, $20 \mathrm{mM} \mathrm{HCl}, 20 \%$ SDS) was added. After incubation over night, the $\mathrm{OD}_{595 \mathrm{~nm}}$ was measured on a Tecan Infinite F50. Medium $\pm 100 \mathrm{nM}$ recombinant ANXA1 or recombinant EGF was renewed daily over the course of the experiment.

\subsection{Cell viability assay (cetuximab)}

Cells were seeded in 96-well plates at a density of 6000 cells per well. The next day, cells were treated with cetuximab (Erbitux ${ }^{\circledR}$, Merck KGaA, Darmstadt, Germany), and cell viability was assessed after the indicated time points by adding $20 \mu \mathrm{l}$ of MTT solution, and after 1-2 $\mathrm{h}$ of incubation, $150 \mu \mathrm{l}$ of solubilisation solution was added to each well. Following overnight incubation, $\mathrm{OD}_{595 \mathrm{~nm}}$ was measured using a Tecan Infinite F50.

\subsection{RNA seq}

Publicly available RNA sequencing data from The Cancer Genome Atlas (TCGA) was used (GSE62944) to compare
ANXA1 gene expression (FPKM values) between healthy and tumour samples from 13 different tissues [39]. The following tissues were used for the study:

\begin{tabular}{lll}
\hline Tissue & Healthy (total) & Tumour (total) \\
\hline Bladder urothelial carcinoma & 19 & 414 \\
Breast invasive carcinoma & 113 & 1119 \\
Colon adenocarcinoma & 41 & 483 \\
Glioblastoma multiforme & 5 & 170 \\
Head and neck squamous carcinoma & 44 & 504 \\
Kidney renal clear cell carcinoma & 72 & 542 \\
Kidney renal papillary cell carcinoma & 32 & 291 \\
Liver hepatocellular carcinoma & 50 & 374 \\
Lung adenocarcinoma & 59 & 541 \\
Lung squamous cell carcinoma & 51 & 502 \\
Prostate adenocarcinoma & 52 & 502 \\
Stomach adenocarcinoma & 37 & 420 \\
Uterine corpus endometrial carcinoma & 35 & 554 \\
\hline
\end{tabular}

The processed FPKM values from healthy and tumour tissues were $\log 2$ transformed and implemented to the Linear Models for Microarray Data (limma) for differential expression analysis [40]. The limma-trend method was used by inputting the $\log 2$-FPKM values into limma's standard pipeline, with trend $=$ TRUE for the empirical Bayes function. Limma uses an eBayes t-test that takes into account the global variance to minimise the influence of noise of local variance for small samples.

\subsection{Immunohistochemistry}

Immunohistochemical staining on head and neck cancer tissue samples was previously approved by the UK National Research Ethics Service (Reference: 10/H0701/ 27). Forty-one consecutive patients with oropharyngeal squamous cell carcinoma were retrospectively identified from pathology databases. Following review by a specialist head and neck pathologist, $4 \mu \mathrm{m}$ paraffin sections were routinely prepared. ANXA1 (Clone MRQ-3), EGFR extracellular domain (Clone 3C6) and EGFR intracellular domain (Clone 5B7) immunohistochemical staining was undertaken using prediluted proprietary kit (Clone MRQ-3, Ventana Medical Systems) on a Ventana Benchmark Autostainer (Ventana Medical Systems) according to manufacturer instructions. Oral squamous cell carcinoma and splenic hairy cell leukaemia were used as positive controls for EGFR and ANXA1, respectively. Omission of the primary antibody served as negative controls.

All cases were independently scored by at least two observers. Where present, the non-dysplastic surface or reticulated crypt epithelium was taken as the referent. An ordinate value of $0-3$ was assigned to the intensity of nuclear staining. The percentage of each intensity was allotted to the entire tumour within the whole mount tissue section and a product of each intensity value and its 
percentage stained within the tumour was determined. A nuclear 'H-Score' was then determined using the following formula: $[(1 \times \%$ cells intensity 1$)+(2 \times \%$ cell intensity 2$)+(3 \times \%$ cells intensity 3$)$ ]. A cytoplasmic ' $\mathrm{H}$ Score' was obtained in a similar way. As it was not possible to differentiate varying intensities of membrane staining, the percentage of any membrane positivity was scored i.e. a binary allocation of 1 (membrane positive cells) or 0 (membrane negative cells) was allotted to the entire tumour within the whole mount tissue section.
Following independent scoring of each case, the differences between each nuclear, cytoplasmic and membrane scores were evaluated. Where a difference in inter-scorer value for each subcellular location was $\geq 50$, an average of the two scores was taken to produce a final score. Where two scores were $>50$ in difference, scorers agreed a consensus value on a multi-headed microscope. This gave a final nuclear, cytoplasmic, and membrane score for each case. The total ANXA1 H-Score for each case was then determined using the final subcellular scores and was
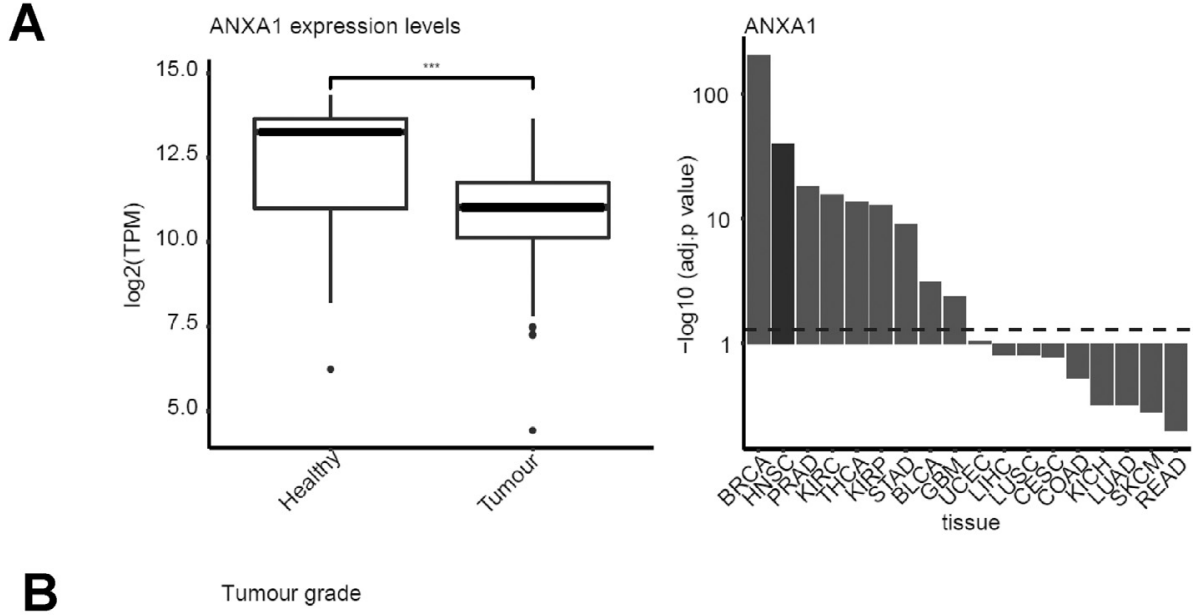

B

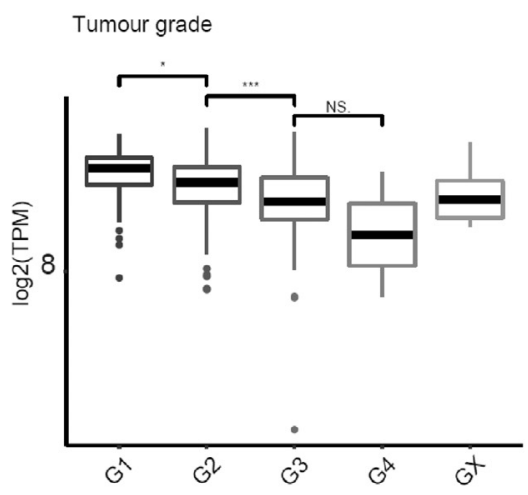

Fig. 1. ANXA1 is downregulated in head and neck cancer, which results in a more oncogenic phenotype in head and neck cancer independently of FPR2 signalling. (A) The FPKM levels of ANXA1 in healthy $(\mathrm{n}=44)$ and tumour $(\mathrm{n}=504)$ samples are shown as boxplots. Median and $25 \%$ and $75 \%$ quantiles are shown as box, $5 \%$ and $95 \%$ quantiles are visualised as whiskers, and outliers are depicted as dots. Bar graphs are representing the $-\log _{10}$ adjusted p-values between tissues of the Cancer Genome Atlas. The p-value for the head and neck squamous cell carcinoma (HNSCC) is represented as a black bar. The number of samples used for the analysis for HNSC were in total 548 samples consisting of $n$ Healthy $=44$, $n$ Tumour $=504$ (p-value $=3.33 \mathrm{e}-12$ ). (B) The clinical variable considering the tumour grade was used to analyse ANXA1 mRNA levels during tumour progression by boxplots. Median and $25 \%$ and $75 \%$ quantiles are shown as box, $5 \%$ and $95 \%$ quantiles are visualised as whiskers and outliers are depicted as dots. The number of samples used for the analysis of the tumour grades were in total 490 samples consisting of $\mathrm{n} \mathrm{G1}=59, \mathrm{n} \mathrm{G} 2=295, \mathrm{n} \mathrm{G} 3=117, \mathrm{n}$ G4 $=2$, $\mathrm{n}$ GX $=17$. Tumour grades that were not identified were removed from the analysis. NS: $\mathrm{p}>0.05, *: \mathrm{p}<0.05, * * *: \mathrm{p}<0.001$. (C-F) ANXA1 is consistently downregulated in head and neck cancer tissue samples. ANXA1 immunohistochemical expression is consistently high in normal oropharyngeal surface (C) and reticulated crypt (D) epithelium (scale bars $=100 \mu \mathrm{m}$ ). By contrast, ANXA1 is consistently downregulated in oropharyngeal squamous cell carcinoma (E: haematoxylin and eosin, F: ANXA1 immunohistochemistry, scale bars $=200 \mu \mathrm{m}$ ). Carcinoma is outlined by black arrows and shown underlining normal surface epithelium. (G) Box and whisker plot of the $\mathrm{H}$ scores obtained for ANXA1 immunohistochemical staining of normal and head and neck cancer tissue samples. Median and 25\% and $75 \%$ quantiles are shown as box, $5 \%$ and $95 \%$ quantiles are visualised as whiskers, and outliers are depicted as dots. $\mathrm{p}<0.0001$, independent $\mathrm{t}$ test. (H) Expression of ANXA1 (full-length and cleaved) was assessed by immunoblotting in sh-scrambled Control (Ctrl) and sh-ANXA1 (KD1) transduced UTSCC6A, H357 and UTSCC34 cell lines. Tubulin served as loading control. The results of one exemplary immunoblot $(\mathrm{n}=3$ ) are depicted. (I) HN5 (left) and HN30 (right) Ctrl as well as ANXA1 KD1 cells were incubated in the absence or presence of $100 \mathrm{nM}$ recombinant ANXA1, and cell proliferation was assessed daily by MTT assay over $5 \mathrm{~d}$. The data represent mean \pm SEM of 3 independent experiments (n.s.: not significant; **: $\mathrm{p} \leq 0.01, * * * *: \mathrm{p} \leq 0.0001,2$-way ANOVA). 

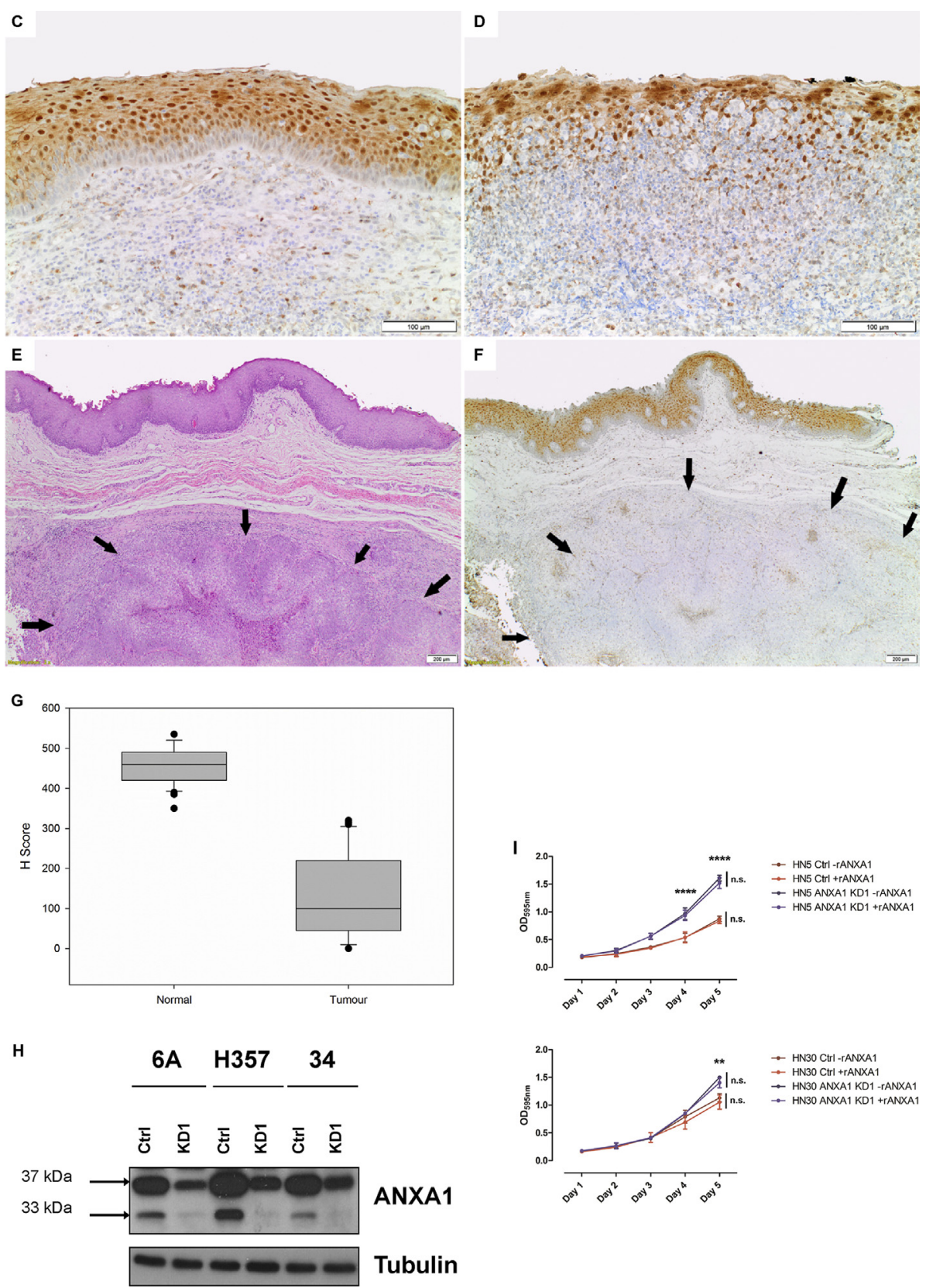

Fig. 1. (continued).

defined as (nuclear H-Score) + (cytoplasmic $\mathrm{H}$ Score $)+(\%$ membrane staining $)$ to give a range of $0-700$.

\subsection{Protein precipitation using trichloracetic acid (TCA)}

Cells were seeded in 6-well plates at a density of 300,000 cells per well. Forty-eight h later, the supernatant of the cells was harvested and centrifuged for $5 \mathrm{~min}$ at $3000 \mathrm{rpm}$. Proteins present in the supernatant were precipitated using TCA as described previously. Briefly, one volume of $100 \%(\mathrm{w} / \mathrm{v})$ TCA (Sigma-Aldrich, St. Louis, MO, USA) was added to 4 volumes of protein sample and incubated on ice for $30 \mathrm{~min}$. Samples were centrifuged for $10 \mathrm{~min}$ at $13,200 \mathrm{rpm}\left(4^{\circ} \mathrm{C}\right)$, and the pellet was washed twice with $500 \mu \mathrm{l}$ ice-cold acetone. Afterwards, the pellet was dried for $5 \mathrm{~min}$ in a heat block $\left(95^{\circ} \mathrm{C}\right), 45 \mu 12 \times$ LSB was subsequently added, and the $\mathrm{pH}$ was restored using $5 \mu 11.5 \mathrm{M}$ Tris, $\mathrm{pH} 8.8$. $25 \mu 1$ of the sample was analysed by immunoblotting. Additionally, the adherent cells were trypsinised, washed once with $1 \times$ PBS, and a whole-cell lysate was prepared. Whole-cell lysate $(40 \mu \mathrm{g})$ was used for immunoblotting.

\subsection{Exosome isolation}

$5 \times 10^{6}$ HN5 Ctrl or ANXA1 knockdown cells were seeded in T75 flasks, in $10 \mathrm{ml}$ medium per flask, 7 flasks 
each. The next day, cells were washed twice with $1 \times$ PBS and once with DMEM w/o FCS. Finally, $10 \mathrm{ml}$ DMEM including $10 \%$ bovine exo-free FBS (as well as pen/strep) were added to each flask. After $48 \mathrm{~h}$, the medium was centrifuged for $20 \mathrm{~min}$ at $300 \times g$ followed by centrifugation at $2000 \times g$ for $20 \mathrm{~min}$ (both: $4{ }^{\circ} \mathrm{C}$ ). The supernatant was stored at $-80^{\circ} \mathrm{C}$ until further analysis. The supernatant was thawed slowly over night at $4{ }^{\circ} \mathrm{C}$, centrifuged at $12200 \times g$ for $45 \min \left(4^{\circ} \mathrm{C}\right)$, and then filtered with a $0.45 \mu \mathrm{m}$ filter followed by ultracentrifugation $(100,000 \times g$ for $2 \mathrm{~h}$ at $4{ }^{\circ} \mathrm{C}$ ). The exosome pellet was sequentially resuspended in sterile $\mathrm{PBS}$ and ultracentrifuged at $100,000 \times g$ for $90 \min \left(4^{\circ} \mathrm{C}\right)$. After this washing step, the exosome pellet was resuspended in $200 \mu 1$ sterile PBS and stored at $-80^{\circ} \mathrm{C}$ until further analysis.

\subsection{Nanoparticle tracking analysis}

Exosome preparations which were stored at $-80{ }^{\circ} \mathrm{C}$ were thawed slowly on ice. A 1:500 dilution (in PBS) was prepared. Nanoparticle tracking analysis (NTA) is based on the principle that the rate of Brownian movement of nanoparticles in solution is a function of their size [41]. In this method, a laser beam is directed at a fixed angle to the vesicle suspension, and the sidescattered light is captured using a standard microscope and high-sensitivity camera. By tracking the Brownian movement of individual nanoparticles over time, a software (NTA 3.1, Malvern, Ltd) calculates their diameter given a known temperature and viscosity of the sample. One advantage of this method is the ability to measure size distribution in polydisperse samples. Exosome analysis was carried out as previously described [42]. Briefly, exosome preparations were diluted to a final volume of $1 \mathrm{ml}$ of $0.45 \mu \mathrm{m}$-filtered sterile PBS and examined using a Nanosight LM10-HS system fitted with an LM14 laser unit-temperature module control (Malvern, Ltd) and with a syringe pump (Harvard Apparatus, Cat No 98-4730) at a constant flow injection of 60 A.U. Individual dilutions were standardised for each cell line and finally used at 1:100-1:200 of exosome preparation over PBS for optimal particle-per-frame performance of NTA. Five video recordings of $30 \mathrm{~s}$ each were captured using automatic laser intensity and camera level per individual sample. Tracking thresholding was manually adjusted for each sample and kept to a minimum value in order to ensure the tracking of all particles and to avoid noise-related artefacts. The videos were analysed, and particle modal size and concentration was calculated. Each condition was repeated in 3 independent experiments.

\subsection{Statistical analysis and software}

Statistical analysis of experimental data was performed using GraphPad Prism 6 software (Graphpad Software Inc., La Jolla, CA, USA), and data were compared using 1-way ANOVA or unpaired student's $t$-test. Data of at least 3 independent experiments are presented as mean \pm standard error of the mean (SEM) unless otherwise stated. $p$-values less than 0.05 were considered statistically significant. The bioinformatic results presented in this article were obtained using $\mathrm{R}$ version 3.3, Bioconductor version 3.3 and the software packages limma 3.28.6.

\section{Results}

\subsection{ANXA1 is downregulated in HNSCC and this mediates an oncogenic phenotype}

We recently showed that ANXA1 is a direct target of the oncogenic microRNA miR-196a in HNSCC cell lines [3]. To determine ANXA1 expression levels in head and neck tumours, RNA Seq data of 504 tumour samples and 44 healthy controls was compared. ANXA1 expression was found significantly downregulated in tumour samples (Fig. 1A). Furthermore, there is a clear correlation between ANXA1 expression and tumour progression from G1 to G4. There is a decrease in ANXA1 in G2 compared to G1, with a further reduction in G3 (Fig. 1B and Supplemental Table 1). To confirm the RNA Seq data, we evaluated ANXA1 protein expression by IHC in 41 oropharyngeal squamous cell carcinoma patient tissue samples. Intrasectional adjacent non-dysplastic surface and reticulated crypt epithelium consistently demonstrated high ANXA1 levels (mean total ANXA1 H score $=455$, median total ANXA1 $\mathrm{H}$ score $=460$; Fig. 1C and D). By contrast, ANXA1 protein levels were consistently decreased in the tumours when compared to intrasectional adjacent non-dysplastic surface and reticulated crypt epithelium $(\mathrm{p}<0.0001$, independent $\mathrm{t}$-test, mean total ANXA1 $\mathrm{H}$ score $=127$, median total ANXA1 $\mathrm{H}$ score $=80$; Fig. $1 \mathrm{E}$ and $\mathrm{F}$ and Supplemental Fig. S1A and B as well as Fig. 1G). Functional studies using shRNA to knockdown ANXA1 resulted in a clear increase in cellular proliferation in 4 different HNSCC cell lines (UTSCC34 and USCC-22B Ctrl and ANXA1, Suppl. S2A and $\mathrm{B}$; HN5 and HN30 Ctrl and ANXA1 knockdown cells, see Fig. 1I). We also attempted to overexpress ANXA1 in HNSCC cell lines using various approaches. Although overexpression of the ANXA1 construct increased the ANXA1 protein levels in 293T cells and GFP as the transfection positive control in HNSCC cell lines was successful, we were unable to overexpress ANXA1 in several HNSCC cell lines (see Suppl. S3A-C). This further supports a growth suppressor role for ANXA1 in HNSCC.

Cleavage and secretion of ANXA1 have recently been reported [16,43]. Extracellular ANXA1 acts as ligand for the formyl peptide receptors FPR1 and FPR2, and 
receptor activation has been associated with increases in cellular proliferation and migration [44]. Immunoblot analysis of the HNSCC cell lines used in this study indicated the expression of a shorter cleaved $33 \mathrm{kDa}$ version of ANXA1 in addition to the full-length $37 \mathrm{kDa}$ protein $($ Fig. $1 \mathrm{H})$. Importantly, the $33 \mathrm{kDa}$, cleaved version of ANXA1 was also greatly diminished in the ANXA1 knockdown cell lines compared to vector
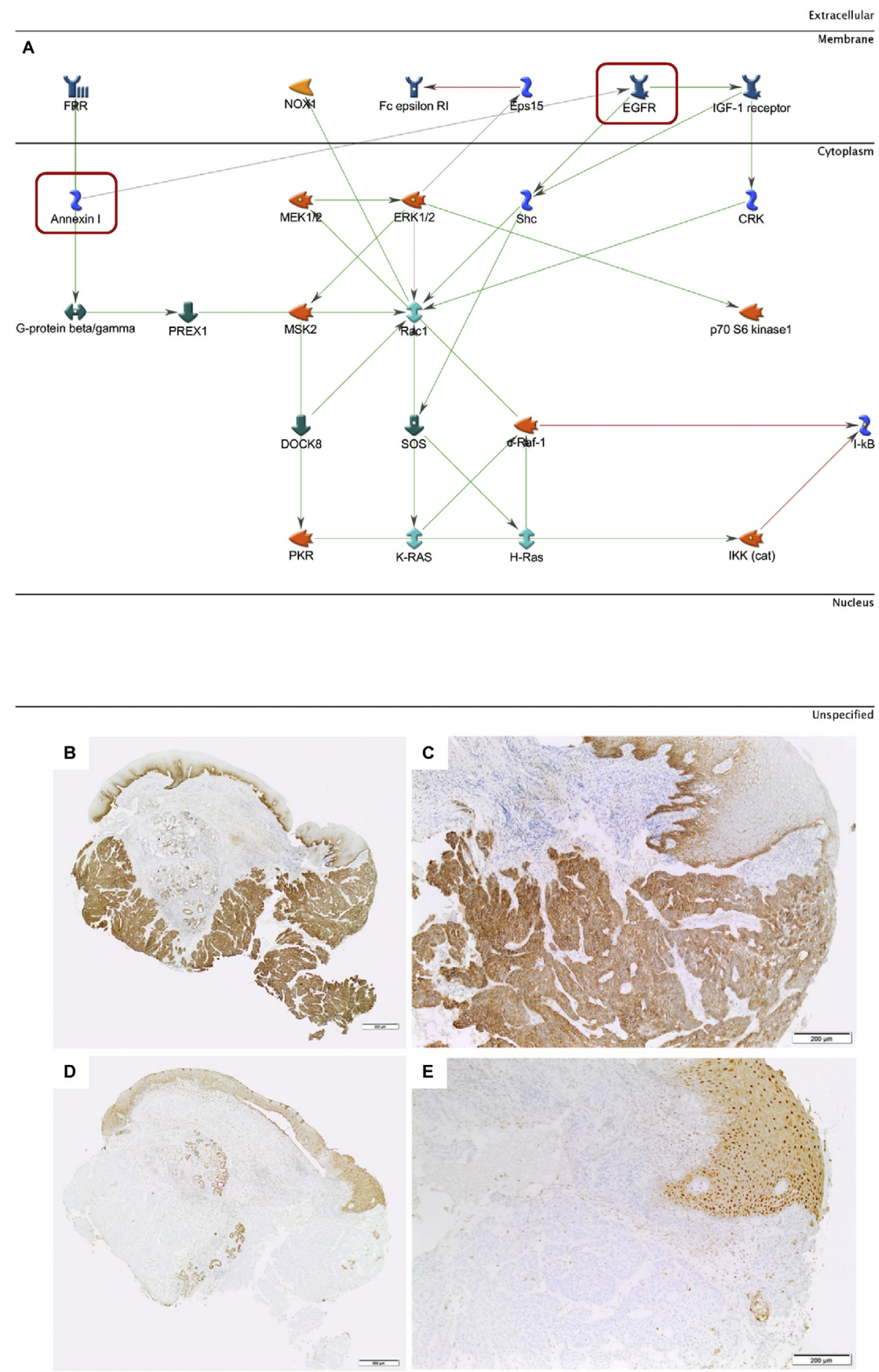

Fig. 2. ANXA1 regulates EGFR signalling. (A) ANXA1 and EGFR network reconstruction based on the literature knowledge obtained from MetaCore ${ }^{\mathrm{TM}}$. (B-E) Representative photomicrograph of intracellular domain of EGFR (B: whole mount view, scale bar $=500 \mu \mathrm{m}$; C: medium power view, scale bar $=200 \mu \mathrm{m}$ ) and ANXA1 (D: whole mount view, scale bar $=500 \mu \mathrm{m}$; E: medium power view, scale bar $=200 \mu \mathrm{m})$ immunohistochemistry in the same head and neck squamous cell carcinoma tissue sample. Non-dysplastic surface epithelium is present at the top $(\mathrm{B}, \mathrm{D})$ and top right $(\mathrm{C}, \mathrm{E})$ while carcinoma is present in the lower part of each photomicrographs. Higher magnifications of $\mathrm{B}$ and $\mathrm{D}$ are shown in $\mathrm{C}$ and $\mathrm{E}$, respectively. 


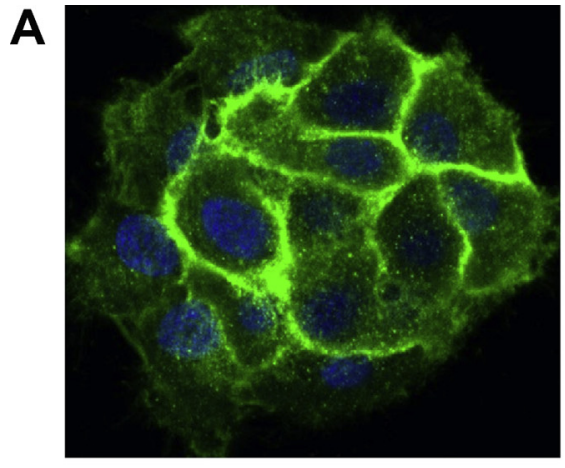

HN5 ANXA1 Ctrl

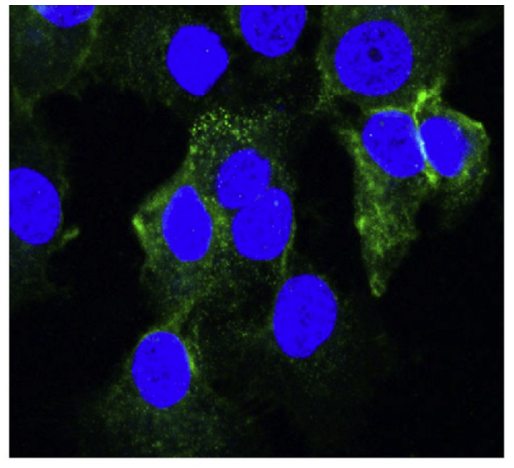

HN5 ANXA1 KD1

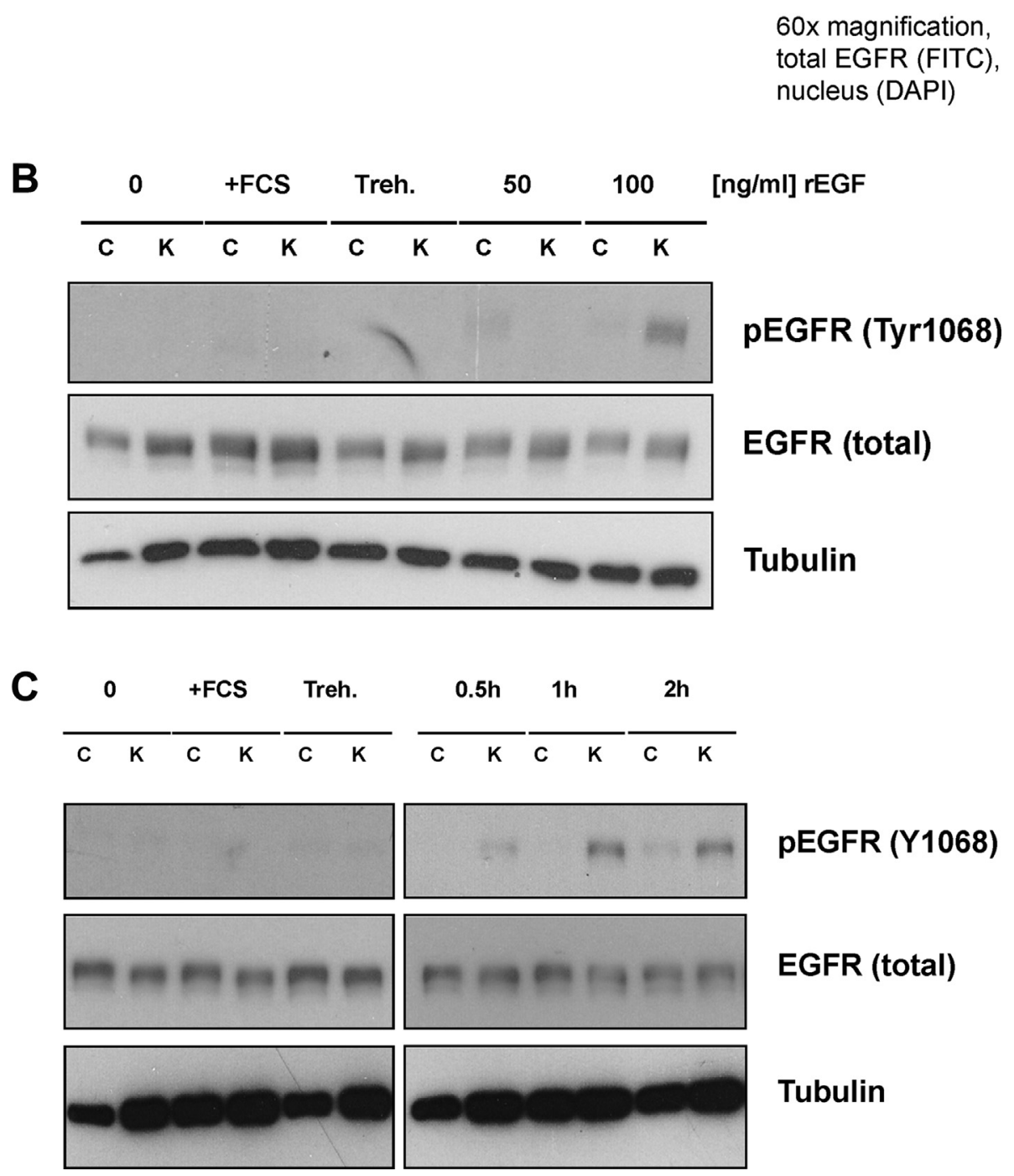

Fig. 3. Downregulation of ANXA1 results in increased and prolonged activation of EGFR signalling. (A) HN5 Ctrl and ANXA1 KD1 cells were stained for total EGFR, and DAPI was used as nuclear marker. Images were taken at $60 \times$ magnification. (B) HN5 Ctrl and ANXA1 KD1 cells were treated with different concentrations of recombinant EGF or 5\% trehalose (Treh) as vehicle control for 30 min, and the levels of total EGFR and EGFR phosphorylated on tyrosine 1068 were analysed by immunoblotting. Tubulin served as loading control. The results of one exemplary immunoblot $(\mathrm{n}=3)$ are depicted. (C) HN5 Ctrl and ANXA1 KD1 cells were incubated in the presence of 100-ng/ml recombinant EGF or 5\% trehalose (Treh) as vehicle control for the indicated time points. Levels of total EGFR and EGFR phosphorylated on tyrosine 1068 were determined by immunoblotting. Tubulin was used as loading control. The results of one exemplary immunoblot $(\mathrm{n}=3)$ are depicted. (D) HN5 Ctrl and ANXA1 KD1 cells were incubated in the presence of $50 \mathrm{ng} / \mathrm{ml}$ recombinant EGF or 5\% trehalose (Treh) as vehicle control for 1 h. Levels of total STAT3, phosphorylated STAT3 (Tyr705), total AKT, 

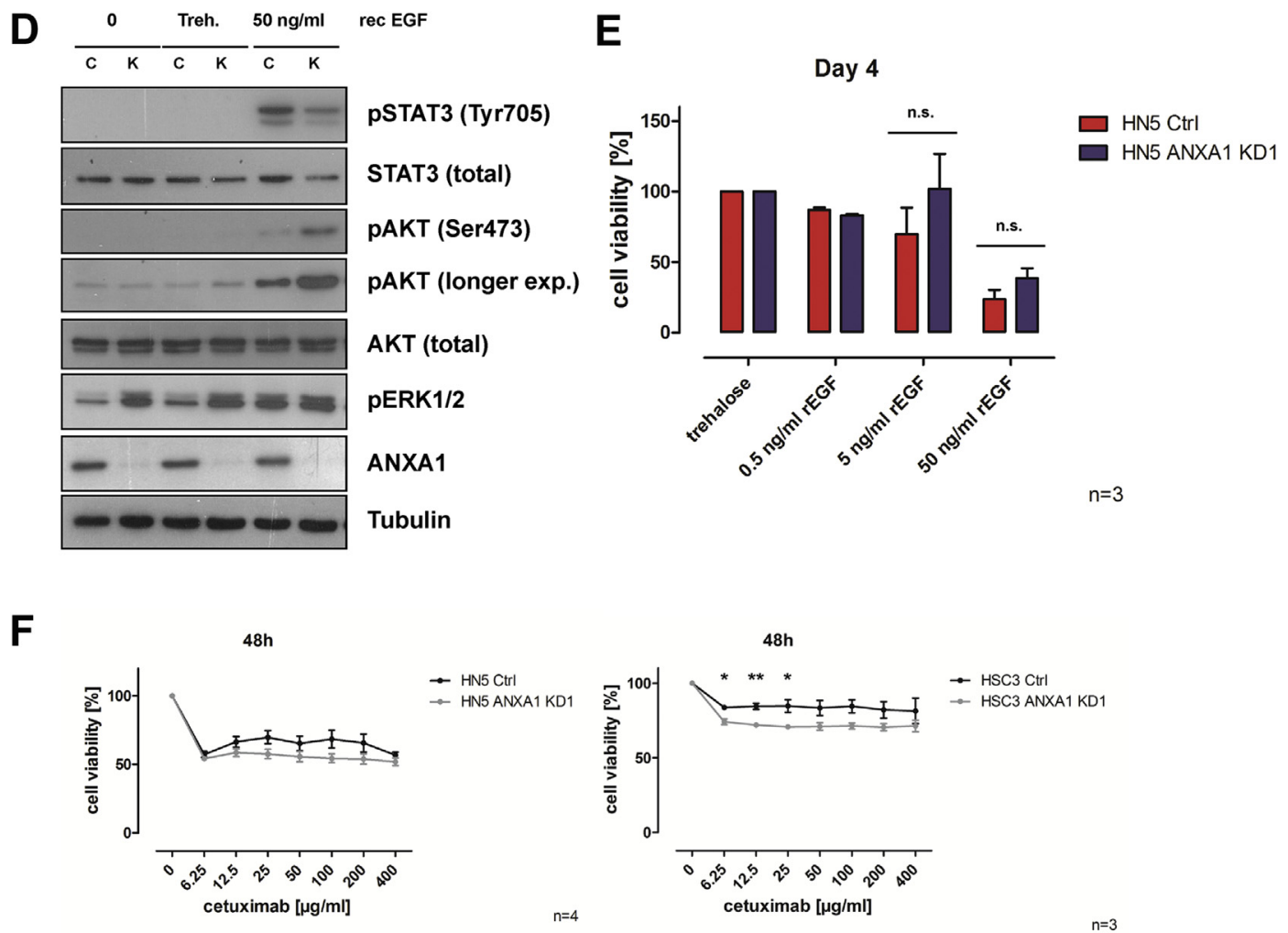

Fig. 3. (continued).

control (Suppl. S4). Although the expression of FPR2 was shown in HN5 and HSC3 by immunofluorescence analysis with MCF-7 cells serving as positive control (Suppl. S5), the HNSCC cell lines showed barely any secretion of ANXA1 into the supernatant (Suppl. S4). Addition of $100 \mathrm{nM}$ recombinant ANXA1 to HN5 or HN30 ANXA1 knockdown and control also did not affect cellular proliferation rates (Fig. 1I). These findings argue against a role of FPR2 signalling and support a cell intrinsic effect of ANXA1 on cellular proliferation in HNSCC.

\subsection{ANXA1 regulates EGFR signalling}

Annexin A1 is mainly known for its anti-inflammatory activities but has also been suggested to influence intracellular signalling pathways. When doing a literature-based network reconstruction using Metacore, a crosstalk between ANXA1 and EGFR signalling was observed (Fig. 2A). EGFR is widely considered to be one of the central drivers of oncogenesis in HNSCC [23]. Head and neck tumour samples were immunohistochemically stained for ANXA1 and EGFR using either an antibody targeting the internal or external domain of the receptor (Fig. 2B-E). Interestingly, an inverse relationship was observed for ANXA1 and EGFR staining. While ANXA1 expression is comparably high in the non-dysplastic area, EGFR expression is low. In contrast to this, the tumour is characterised by high levels of EGFR and almost undetectable ANXA1 expression. Correlation analysis confirmed the trend towards an inverse correlation between (nuclear) ANXA1 and EGFR in the head and neck tumour samples analysed in this study. The correlation was seen for 2 different antibodies targeting the external or internal domain of EGFR (adjusted $r$ squared for EGFR $_{\text {external }}=0.1869934$ and for EGFR internal $=$ 0.1665316) (Suppl. Fig. S6). Furthermore, ANXA1 knockdown indicated a change in the subcellular distribution of EGFR detected by immunofluorescence: the

phosphorylated AKT (Ser473), phosphorylated ERK1/2 as well as Annexin A1 were determined by immunoblotting. Tubulin served as loading control. The results of one exemplary immunoblot $(n=3)$ are depicted. (E) HN5 Ctrl and ANXA1 KD1 cells were incubated in the presence of $0.5,5$ or $50 \mathrm{ng} / \mathrm{ml}$ recombinant EGF with daily medium change. Cell viability was assessed $4 \mathrm{~d}$ later by MTT assay. Data were normalised to vehicle control (trehalose) and the mean \pm SEM of 3 independent experiments is shown (n.s.: not significant, 2-way ANOVA). (F) Cell viability of HN5 and HSC3 Ctrl as well as ANXA1 KD1 cells in the presence of increasing concentrations of cetuximab was assessed by MTT cell viability assay after the indicated time points. Data represent mean \pm SEM of 3 (HSC3) or 4 (HN5) independent experiments. Differences observed in cell viability between Ctrl and ANXA1 knockdown cells were statistically analysed and $\mathrm{p}$-values are indicated (unpaired t-test; $* \mathrm{p}<0.05, * *: \mathrm{p}=0.005$ ). 
A

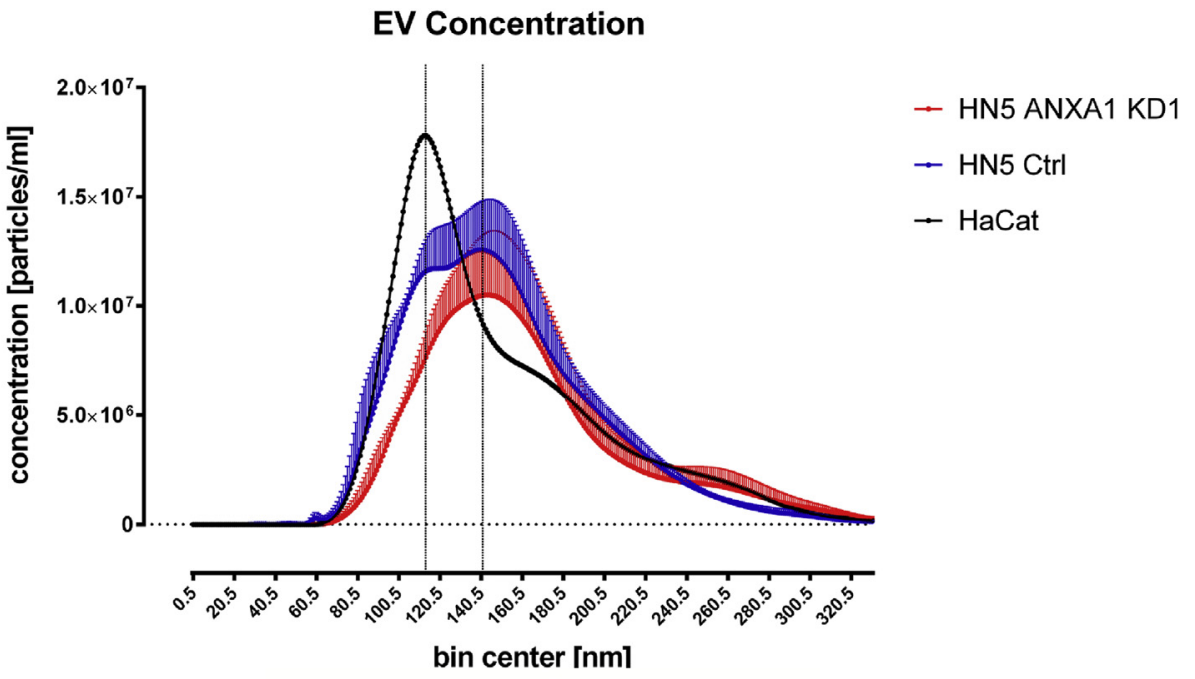

B

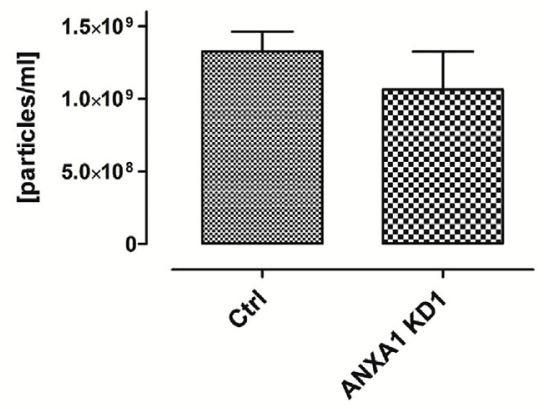

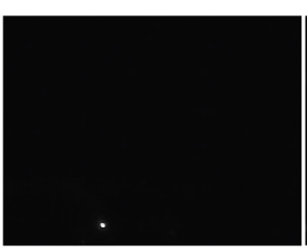

vehicle

C

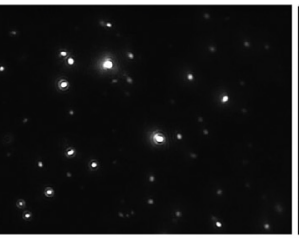

HN5 Ctrl

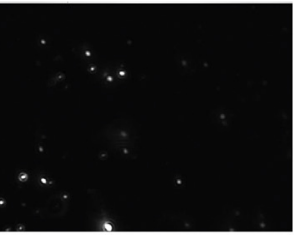

HN5 ANXA1 KD1

pEGFR (Tyr1068)

CD63

$1 \quad 0.61$

(pEGFR/CD63) 
staining pattern for EGFR in ANXA1 knockdown cells was more diffuse, and there was a clear decrease in membraneous EGFR compared to control cells (Fig. 3A). This hints at increased activation and intracellular trafficking of EGFR in ANXA1 knockdown cells.

We then studied the response of the ANXA1 knockdown cells towards EGFR stimulation. When ANXA1 knockdown and control cells were incubated for $30 \mathrm{~min}$ in the presence of 50 or $100 \mathrm{ng} / \mathrm{ml} \mathrm{recombi-}$ nant EGF, there was an increase in EGFR tyrosine 1068 phosphorylation in ANXA1 knockdown cells (Fig. 3B). This increase in EGFR tyrosine 1068 phosphorylation was stable over time and was maintained after 1 and $2 \mathrm{~h}$ of receptor stimulation with $100 \mathrm{ng} / \mathrm{ml}$ recombinant EGF (Fig. 3C). This indicates an increased and/or prolonged activation of EGFR receptor signalling in ANXA1 knockdown cells. EGFR signalling is known to control several different cellular signalling pathways such as PI3 kinase/AKT, ERK and JAK/STAT signalling pathways [30]. Phosphorylation and activation of STAT3 and AKT was observed in the presence of recombinant EGF in both Ctrl and ANXA1 knockdown cells. However, ANXA1 knockdown cells showed increased phosphorylation of AKT at serine 473 after receptor stimulation indicating increased activation of the PI3 kinase/AKT pathway in these cells whereas STAT3 signalling via STAT3 phosphorylation was reduced (Fig. 3D). Of note, increased phosphorylation of ERK1/2 was already observed in the absence of stimulus in ANXA1 knockdown cells, which did not further increase in response to EGF treatment indicating EGF-independent ERK1/2 activation by ANXA1 downregulation. By contrast, control cells exhibited low baseline levels of phosphorylated ERK1/2, and phosphorylation was upregulated in the presence of $50 \mathrm{ng} / \mathrm{ml}$ recombinant EGF (Fig. 3D). It has already been reported that EGF inhibits the growth of HN5 cells and can induce senescence [45]. We confirmed the inhibitory effect of EGF on wild-type HN5 cells by MTT assay; however, ANXA1 knockdown HN5 cells had the tendency to show increased survival in the presence of 5 and $50 \mathrm{ng} / \mathrm{ml}$ recombinant EGF (Fig. 3E) again suggesting a regulation of the EGFR pathway by ANXA1.

Cetuximab, an IgG1 chimerised $\alpha$ EGFR monoclonal antibody, binds with high affinity to the extracellular EGF domain of EGFR and thereby prevents ligand binding and receptor activation. ANXA1 knockdown and control cells were treated with increasing concentrations of cetuximab, and cell viability was assessed by MTT assay. Interestingly, there was a trend towards increased cetuximab sensitivity in HN5 and HSC3 cells when ANXA1 was downregulated indicating a potential EGFR-dependent response (Fig. 3F).

\subsection{ANXA1 protein levels affect exosome production and the release of exosomal phospho-EGFR}

Recent findings have demonstrated an important role of exosomal EGFR in metastasis formation. As ILVs can be precursors of exosomes, we speculated that ANXA1 may regulate exosomal EGFR release $[35,36]$. Extracellular vesicles of HN5 Ctrl and ANXA1 knockdown cells were isolated by ultracentrifugation and characterised by nanoparticle tracking analysis. When measuring the concentration of particles per $\mathrm{ml}$ in relation to the diameter of the particles in $\mathrm{nm}$, there was a difference in the distribution pattern of the particles between the HN5 Ctrl and ANXA1 knockdown cells (Fig. 4A). Particles of HaCat cells which are known to secret high levels of exosomal EGFR served as positive control, and the size of their exosomes peaked around $115 \mathrm{~nm}$ [46]. Interestingly, while exosomes of HN5 Ctrl cells showed a bimodal size distribution with peaks at approximately $115 \mathrm{~nm}$ and $140.5 \mathrm{~nm}$, the peak at $115 \mathrm{~nm}$ was lost in HN5 ANXA1 knockdown cells (Fig. 4A). Combined with the observation that HN5 ANXA1 knockdown cells tended to produce lower levels of exosomes per ml (Fig. 4B), this indicates a difference in exosome biogenesis and cargo release in ANXA1-modulated cells. To investigate this in more detail, the amount of exosomal phosphorylated EGFR in these cells was analysed by immunoblotting. Downregulation of ANXA1 resulted in reduced levels of exosomal phosphorylated EGFR (Fig. 4C). This further supports an involvement of ANXA1 in cell-cell communication via exosomal EGFR.

\section{Discussion}

Current treatment strategies for head and neck cancer are surgery and radiotherapy alone or in combination with chemotherapy. These strategies are ineffective with up to $50 \%$ of patients relapsing and are associated with significant morbidity [38]. This highlights the need for better predictive biomarkers and novel, more specific

Fig. 4. Downregulation of ANXA1 changes exosome production levels and exosomal phospho-EGFR content. (A) Extracellular vesicles (EV) from cell culture supernatant of HN5 Ctrl, ANXA1 KD1 and HaCat cell lines were isolated by ultracentrifugation and analysed by nanoparticle tracking using Nanosight. The concentration of particles per $\mathrm{ml}$ was plotted against their bin centre in $\mathrm{nm}$. Data represent mean \pm SEM of 3 independent experiments. (B) The overall concentration of isolated EV of HN5 Ctrl and ANXA1 KD1 cell culture supernatant was determined in the course of nanoparticle tracking using Nanosight. Data represent particles per ml and depict mean \pm SEM of 3 independent experiments (not significant, unpaired t-test, $p=0.4268$ ). Video stills of exemplary measurements of exosomes in the vehicle, HN5 Ctrl and ANXA1 KD1 are shown below the bar chart. (C) Levels of tyrosine 1068 phospho-EGFR and exosomal marker CD63 in the extracellular vesicles of HN5 Ctrl and ANXA1 KD1 cells were determined by immunoblotting ( $\mathrm{n}=3$ ). 
treatment approaches. We recently demonstrated a link between oncogenic miR-196a and poor treatment response in HNSCC patients. Further studies identified ANXA1 as a direct target of the oncogenic microRNA miR-196a potentially mediating therapy outcome [3]. In this study we demonstrate a tumour suppressor role of ANXA1 in HNSCC. ANXA1 was consistently downregulated in the analysed expression profiling data sets and its expression levels negatively correlated with the number of lymph nodes involved as well as the pathology stage $\mathrm{N}$ as shown by Spearman correlation test. Immunohistochemical staining of patient tumour samples detected substantially less ANXA1 in tumour samples compared to adjacent normal tissue. Overexpression of ANXA1 has been reported to have various growth inhibitory effects such as cell cycle arrest and/or apoptotic cell death in a range of cell lines through various mechanisms [47-50]. We were unable to establish cell lines which transiently or stably overexpressed ANXA1 despite using various techniques. This suggests that the forced ANXA1 expression in HNSCC cell lines induces a selective cell survival disadvantage and consequently loss of the overexpressing clones. The results further support a tumour suppressive function of ANXA1 in HNSCC.

The precise mechanism of ANXA1 downregulation in HNSCC remains currently unclear. As mentioned above, we have found ANXA1 to be a target for downregulation by miR-196a in HNSCC. Although ANXA1 mutations are a rare event in HNSCC, one of the mutations reported (at amino acid position 122) is a nonsense mutation resulting in a truncated ANXA1 (http://www.tumorportal.org/view? geneSymbol=ANXA1,https://hive.biochemistry.gwu.ed u/cgi-bin/prd/biomuta/servlet.cgi?gpageid $=11$ \&searchfie $1 \mathrm{~d} 1=$ gene_name\&searchvalue $1=$ ANXA1). Additionally, chewing tobacco, which is one of the main risk factors of oral cancer, results in the loss of the anti-inflammatory activity of ANXA1 [20]. Collectively, these findings identify ANXA1 as a potential predictive and prognostic biomarker in HNSCC.

We have previously demonstrated that ANXA1 knockdown in HNSCC cell lines induces increased tumour invasion and elevates the expression of EMT markers [3]. ANXA1 is mainly an anti-inflammatory protein with important roles in the innate and adaptive immune response [7]. Inflammation has been shown to alter the tumour microenvironment as well as tumour immune responses and to induce tumour hypoxia [51,52]. Additionally, hypoxia induces a chronic inflammatory state which further reduces immune responses within the tumour microenvironment [53,54]. Considering the crucial role of ANXA1 in controlling inflammation, it would be important to investigate whether downregulation of ANXA1 in HNSCC results in increased tumour hypoxia and more aggressive disease as well as therapy resistance in HNSCC. This is particularly relevant as we initially identified miR-196a, which targets ANXA1, in HNSCC tumours with poor treatment outcome which is also a hallmark of more hypoxic cancers [3].

ANXA1 seems to have both pro- or anti-proliferative activity depending on tumour types. Downregulation of ANXA1 in oropharyngeal cancer as well as other cancer subtypes has been described [15,55-58]. Surprisingly, ANXA1 null mice show impaired tumour growth, and specific subsets of breast cancers show upregulation of ANXA1 [17,59]. The differences in ANXA1 activity could be determined by different subcellular locations, nuclear and membranous, and/or to its secreted and intracellular forms [13]. So far, the tumour-associated functions of ANXA1 have been mainly linked to a paracrine/autocrine mechanism via secretion of ANXA1 and its interaction with its receptor FPR 1 and/or 2 on the cell surface [60]. However, in this study, no significant levels of the secreted form of ANXA1 were detected in the HNSCC cell lines analysed. Our data therefore do not support a major contribution of extracellular ANXA1 and paracrine/autocrine signalling pathways in controlling cellular proliferation in HNSCC. The findings here suggest that the intracellular form of ANXA1 and its cell autonomous effects on intracellular signalling pathways may be important in regulating HNSCC tumour cell growth and invasion [3]. One candidate signalling pathway could be ERK1/2-mediated signalling as we detected constitutively high levels of phosphorylated ERK in the ANXA1 knockdown HNSCC cells as compared to control cells. Interestingly, $\mathrm{He}$ et al. recently showed that ANXA1 can decrease the nuclear accumulation of phosphorylated ERK, which leads to reduced expression of cyclin D1 and subsequent inhibition of proliferation of rat pulmonary arterial smooth muscle cells [48]. We also observed a decrease in STAT3 phosphorylation and thus STAT3 pathway activation in the absence of ANXA1. Changes in ANXA1 expression levels have already been reported to affect STAT3 activation positively or negatively depending on the type of cancer. Silencing of another annexin superfamily member, Annexin A2, has additionally been associated with reduced EGF-induced STAT3 phosphorylation [61-63].

ANXA1 has been reported to regulate EGFRdependent signalling pathways [64]. Upregulation of EGFR and increased EGFR phosphorylation is the most common oncogenic event in HNSCC $[65,66]$. Elevated EGFR expression as well as gene amplification has been shown to correlate with poor prognosis in HNSCC and a variety of other cancer types such as gastric, oesophageal, lung and triple negative breast cancers [67-70]. Increased expression levels of EGFR correlate with chemoresistance while lentiviral or siRNA-mediated downregulation of EGFR expression has been reported to enhance chemosensitivity in HNSCC [71-73]. In recent years, multiple EGFR inhibitors have been developed, but cancer cells show select sensitivity to these drugs. The inherent and/or 
acquired resistance of cancers to EGFR inhibitors is unclear and is under intense investigation. Mutations in EGFR gene or changes in the subcellular localisation of EGFR, resulting in inaccessibility of the inhibitor to the receptor, could be a contributing factor. However, most studies have focused on the cell autonomous EGFR regulation as a cause of treatment resistance. Interestingly, non-cell autonomous factors can also affect sensitivity towards EGFR-targeting drugs such as cetuximab. Van Dommelen et al., for example, reported an association between cetuximab response and extracellular vesicles [74]. It is known that EGFR signalling is highly regulated by intracellular trafficking, and molecules involved in this process can affect EGFR signalling and EGFR localisation [32,75]. To this end, Annexin A1 has shown to be required for the formation of ILVs in multivesicular bodies (MVBs) that sequester ligandbound EGFR away from the limiting membrane [21]. In this study, we show that downregulation of ANXA1 resulted in increased and sustained phosphorylation of EGFR after EGF stimulation and that there was an inverse correlation between ANXA1 and EGFR expression levels in head and neck tumour samples. Interestingly, sustained phosphorylation of EGFR at tyrosine 1173,845 , and/or 1068 has been reported to correlate with poor prognosis in HNSCC, and high levels of EGFR phosphorylation facilitate metastasis in salivary gland carcinomas [76-78]. Downregulation of ANXA1 in combination with increased EGFR levels and EGFR-dependent signalling could therefore contribute to a malignant phenotype in HNSCC driving cancer progression and invasion. Our data suggest that one mechanism by which ANXA1 mediates its tumour suppressive activities in healthy cells is via ensuring ordered and timely degradation of phosphorylated EGFR and safeguarding regulated EGFR signalling pathway.

Given the role of ANXA1 in ILV formation and the reports that ILVs are precursors of exosomes, we speculated that ANXA1 could also play a role in exosome production in addition to its role in EGFR signalling termination $[33,79]$. Here, we observed a reduction in exosome production in HNSCC cell lines in response to ANXA1 knockdown. Interestingly, decreased levels of ANXA1 were also associated with disease progression. To our knowledge, this is one of the first reports which links disease progression and decreased activity of a potential tumour suppressor (ANXA1) with reduced exosome levels. So far, malignant cells and disease progression have generally been associated with increased exosome production $[46,80]$. Furthermore, our findings indicate that it is rather specific cargo and not general exosome levels that could be decisive for tumour progression. Along these lines, we see the disappearance of a subpopulation of exosomes in the analysed ANXA1 knockdown cells and an accompanying decrease in the amount of exosomal phospho-EGFR. This hints at the existence of different exosome biogenesis pathways.
ANXA1 might be responsible for the recruitment of phospho-EGFR into exosomes, and this might be mediated by an (transient) interaction of ANXA1 and phospho-EGFR which has already been reported [33]. Exosomal EGFR has been linked to organ-specific metastasis by influencing premetastatic niche formation and to tumour immunity by inducing tolerance in recipient dendritic cells and as a consequence tumour antigen-specific regulatory $\mathrm{T}$ cells $[36,37]$. In summary, ANXA1 seems to be located at the interface of inflammation and cancer. The fact that cancer is considered as 'wounds that do not heal' highlights the importance of balanced levels of anti-inflammatory proteins such as ANXA1 [81]. Future studies will be important to determine the impact of ANXA1-mediated reduction of exosomal phospho-EGFR on tumour microenvironment, hypoxia and tumour immunity.

\section{Conclusion}

Our studies support a tumour suppressive function of ANXA1 in head and neck cancer. This role can mainly be attributed to the intracellular version of the protein. Intracellular ANXA1 seems to exert its activities cell autonomously by affecting the strength and duration of EGFR-dependent signalling events but also by regulating exosomal phospho-EGFR release and thereby cell-cell communication. Further studies into the role of ANXA1 in exosome production in general and exosomal phospho-EGFR recruitment are required to fully elucidate the ANXA1/EGFR axis and its importance in HNSCC pathogenesis.

\section{Conflict of interest statement}

None declared for all authors.

\section{Acknowledgement}

The authors would like to thank Prof Mauro Perretti, Queen Mary University of London, for recombinant Annexin A1 and FPR2 antibody, and Dr. Stéphane Gobeil, CHUL Research Centre, Laval University, Canada for lentiviral ANXA1 shRNA and control. This study was supported by a grant from the Rosetrees Trust and Stoneygate Trust, Ref: M117-F1.

\section{Appendix A. Supplementary data}

Supplementary data related to this article can be found at https://doi.org/10.1016/j.ejca.2018.07.123.

\section{References}

[1] Shield KD, Ferlay J, Jemal A, Sankaranarayanan R, Chaturvedi AK, Bray F, et al. The global incidence of lip, oral 
cavity, and pharyngeal cancers by subsite in 2012. CA Cancer J Clin 2017;67(1):51-64.

[2] Felippu AW, Freire EC, de Arruda Silva R, Guimarães AV, Dedivitis RA. Impact of delay in the diagnosis and treatment of head and neck cancer. Braz J Otorhinolaryngol 2016;82(2):140-3.

[3] Suh YE, Raulf N, Gäken J, Lawler K, Urbano TG, Bullenkamp J, et al. MicroRNA-196a promotes an oncogenic effect in head and neck cancer cells by suppressing annexin A1 and enhancing radioresistance. Int J Cancer 2015;137(5): 1021-34.

[4] Moss SE, Morgan RO. The annexins. Gen Biol 2004;5(4):219.

[5] Rosengarth A, Gerke V, Luecke H. X-ray structure of full-length annexin 1 and implications for membrane aggregation. J Mol Biol 2001;306(3):489-98.

[6] Rescher U, Gerke V. Annexins-unique membrane binding proteins with diverse functions. J Cell Sci 2004;117(Pt 13):2631-9.

[7] Gavins FN, Hickey MJ. Annexin A1 and the regulation of innate and adaptive immunity. Front Immunol 2012;3:354.

[8] Belvedere R, Bizzarro V, Forte G, Dal Piaz F, Parente L, Petrella A. Annexin A1 contributes to pancreatic cancer cell phenotype, behaviour and metastatic potential independently of formyl peptide receptor pathway. Sci Rep 2016;6:29660.

[9] McNeil AK, Rescher U, Gerke V, McNeil PL. Requirement for annexin A1 in plasma membrane repair. J Biol Chem 2006; 281(46):35202-7.

[10] Kang JH, Li M, Chen X, Yin XM. Proteomics analysis of starved cells revealed Annexin A1 as an important regulator of autophagic degradation. Biochem Biophys Res Commun 2011;407(3): $581-6$.

[11] Bandorowicz-Pikula J, Wos M, Pikula S. Do annexins participate in lipid messenger mediated intracellular signaling? A question revisited. Mol Membr Biol 2012;29(7):229-42.

[12] Solito E, Christian HC, Festa M, Mulla A, Tierney T, Flower RJ, et al. Post-translational modification plays an essential role in the translocation of annexin A1 from the cytoplasm to the cell surface. FASEB J 2006;20(9):1498-500.

[13] Boudhraa Z, Bouchon B, Viallard C, D'Incan M, Degoul F. Annexin A1 localization and its relevance to cancer. Clin Sci (Lond) 2016;130(4):205-20.

[14] Szanto I, Mark L, Bona A, Maasz G, Sandor B, Gelencser G, et al. High-throughput screening of saliva for early detection of oral cancer: a pilot study. Technol Cancer Res Treat 2012;11(2): $181-8$.

[15] Zhu DW, Yang X, Yang CZ, Ma J, Liu Y, Yan M, et al. Annexin A1 down-regulation in oral squamous cell carcinoma correlates to pathological differentiation grade. Oral Oncol 2013;49(6):542-50.

[16] Belvedere R, Bizzarro V, Popolo A, Dal Piaz F, Vasaturo M, Picardi P, et al. Role of intracellular and extracellular annexin A1 in migration and invasion of human pancreatic carcinoma cells. BMC Cancer 2014;14:961.

[17] Sobral-Leite M, Wesseling J, Smit VT, Nevanlinna H, van Miltenburg MH, Sanders J, et al. Annexin A1 expression in a pooled breast cancer series: association with tumor subtypes and prognosis. BMC Med 2015;13:156.

[18] Calmon MF, Sichero L, Boccardo E, Villa LL, Rahal P. HPV16 E6 regulates annexin 1 (ANXA1) protein expression in cervical carcinoma cell lines. Virology 2016;496:35-41.

[19] Lin Y, Lin G, Fang W, Zhu H, Chu K. Increased expression of annexin Al predicts poor prognosis in human hepatocellular carcinoma and enhances cell malignant phenotype. Med Oncol 2014;31(12):327.

[20] Vishwanatha JK, Swinney R, Banerjee AG. Modulation of annexin I and cyclooxygenase-2 in smokeless tobacco-induced inflammation and oral cancer. Mol Cell Biochem 2003; 248(1-2):67-75.

[21] Poeter M, Radke S, Koese M, Hessner F, Hegemann A, Musiol A, et al. Disruption of the annexin A1/S100A11 complex increases the migration and clonogenic growth by dysregulating epithelial growth factor (EGF) signaling. Biochim Biophys Acta 2013;1833(7):1700-11.

[22] Normanno N, De Luca A, Bianco C, Strizzi L, Mancino M, Maiello MR, et al. Epidermal growth factor receptor (EGFR) signaling in cancer. Gene 2006;366(1):2-16.

[23] Mayer A, Zahnreich S, Brieger J, Vaupel P, Schmidberger H. Downregulation of EGFR in hypoxic, diffusion-limited areas of squamous cell carcinomas of the head and neck. Br J Cancer 2016; 115(11):1351-8.

[24] Maiti GP, Mondal P, Mukherjee N, Ghosh A, Ghosh S, Dey S, et al. Overexpression of EGFR in head and neck squamous cell carcinoma is associated with inactivation of SH3GL2 and CDC25A genes. PLoS One 2013;8(5):e63440.

[25] Alorabi M, Shonka NA, Ganti AK. EGFR monoclonal antibodies in locally advanced head and neck squamous cell carcinoma: What is their current role? Crit Rev Oncol Hematol 2016;99:170-9.

[26] Yoshida K, Kyo E, Tsuda T, Tsujino T, Ito M, Niimoto M, et al. EGF and TGF-alpha, the ligands of hyperproduced EGFR in human esophageal carcinoma cells, act as autocrine growth factors. Int J Cancer 1990;45(1):131-5.

[27] Singh B, Carpenter G, Coffey RJ. EGF receptor ligands: recent advances. F1000Res 2016;5.

[28] Guo G, Gong K, Wohlfeld B, Hatanpaa KJ, Zhao D, Habib AA. Ligand-independent EGFR signaling. Cancer Res 2015;75(17): $3436-41$.

[29] Chakraborty S, Li L, Puliyappadamba VT, Guo G, Hatanpaa KJ, Mickey B, et al. Constitutive and ligand-induced EGFR signalling triggers distinct and mutually exclusive downstream signalling networks. Nat Commun 2014;5:5811.

[30] Chong CR, Janne PA. The quest to overcome resistance to EGFR-targeted therapies in cancer. Nat Med 2013;19(11): $1389-400$.

[31] Baselga J. The EGFR as a target for anticancer therapy-focus on cetuximab. Eur J Cancer 2001;37(Suppl. 4):S16-22.

[32] Tan X, Lambert PF, Rapraeger AC, Anderson RA. Stressinduced EGFR trafficking: mechanisms, functions, and therapeutic implications. Trends Cell Biol 2016;26(5):352-66.

[33] Eden ER, Sanchez-Heras E, Tsapara A, Sobota A, Levine TP, Futter CE. Annexin A1 tethers membrane contact sites that mediate ER to endosome cholesterol transport. Dev Cell 2016; 37(5):473-83.

[34] White IJ, Bailey LM, Aghakhani MR, Moss SE, Futter CE. EGF stimulates annexin 1-dependent inward vesiculation in a multivesicular endosome subpopulation. EMBO J 2006;25(1):1-12.

[35] Gutiérrez-Vázquez C, Villarroya-Beltri C, Mittelbrunn M, Sánchez-Madrid F. Transfer of extracellular vesicles during immune cell-cell interactions. Immunol Rev 2013;251(1):125-42.

[36] Zhang H, Deng T, Liu R, Bai M, Zhou L, Wang X, et al. Exosome-delivered EGFR regulates liver microenvironment to promote gastric cancer liver metastasis. Nat Commun 2017 Apr 10;8: 15016.

[37] Huang SH, Li Y, Zhang J, Rong J, Ye S. Epidermal growth factor receptor-containing exosomes induce tumor-specific regulatory $\mathrm{T}$ cells. Cancer Investig 2013;31(5):330-5.

[38] Raulf N, El-Attar R, Kulms D, Lecis D, Delia D, Walczak H, et al. Differential response of head and neck cancer cell lines to TRAIL or Smac mimetics is associated with the cellular levels and activity of caspase-8 and caspase-10. Br J Cancer 2014;111(10): 1955-64.

[39] Rahman M, Jackson LK, Johnson WE, Li DY, Bild AH, Piccolo SR. Alternative preprocessing of RNA-sequencing data in the cancer genome atlas leads to improved analysis results. Bioinformatics 2015;31(22):3666-72.

[40] Ritchie ME, Phipson B, Wu D, Hu Y, Law CW, Shi W, et al. Limma powers differential expression analyses for RNA-sequencing and microarray studies. Nucleic Acids Res 2015;43(7):e47.

[41] Dragovic RA, Gardiner C, Brooks AS, Tannetta DS, Ferguson DJ, Hole P, et al. Sizing and phenotyping of cellular 
vesicles using nanoparticle tracking analysis. Nanomedicine (Lond) 2011;7(6):780-8.

[42] Vicencio JM, Yellon DM, Sivaraman V, Das D, Boi-Doku C, Arjun $\mathrm{S}$, et al. Plasma exosomes protect the myocardium from ischemia-reperfusion injury. J Am Coll Cardiol 2015;65(15): $1525-36$.

[43] Zhu F, Xu C, Jiang Z, Jin M, Wang L, Zeng S, et al. Nuclear localization of annexin Al correlates with advanced disease and peritoneal dissemination in patients with gastric carcinoma. Anat Rec (Hoboken) 2010;293(8):1310-4.

[44] Cheng TY, Wu MS, Lin JT, Lin MT, Shun CT, Huang HY, et al. Annexin A1 is associated with gastric cancer survival and promotes gastric cancer cell invasiveness through the formyl peptide receptor/extracellular signal-regulated kinase/integrin beta-1binding protein 1 pathway. Cancer 2012;118(23):5757-67.

[45] Modjtahedi H, Styles J, Dean C. The growth-response of human tumor-cell lines expressing the EGF receptor to treatment with EGF and or mabs that block ligand-binding. Int J Oncol 1993; 3(2):237-43.

[46] Perez-Torres M, Valle BL, Maihle NJ, Negron-Vega L, NievesAlicea R, Cora EM. Shedding of epidermal growth factor receptor is a regulated process that occurs with overexpression in malignant cells. Exp Cell Res 2008;314(16):2907-18.

[47] Yuan Y, Anbalagan D, Lee LH, Samy RP, Shanmugam MK, Kumar AP, et al. ANXA1 inhibits miRNA-196a in a negative feedback loop through NF-kB and c-Myc to reduce breast cancer proliferation. Oncotarget 2016;7(19):27007-20.

[48] He J, Yi B, Chen Y, Huang Q, Wang H, Lu K, et al. The ET-1mediated carbonylation and degradation of ANXA1 induce inflammatory phenotype and proliferation of pulmonary artery smooth muscle cells in HPS. PLoS One 2017;12(4):e0175443.

[49] Wan YM, Tian J, Qi L, Liu LM, Xu N. ANXA1 affects cell proliferation, invasion and epithelial-mesenchymal transition of oral squamous cell carcinoma. Exp Ther Med 2017;14(5):5214-8.

[50] Lim LH, Pervaiz S. Annexin 1: the new face of an old molecule. FASEB J 2007;21(4):968-75.

[51] Han Y, Cho U, Kim S, Park IS, Cho JH, Dhanasekaran DN, et al. Tumour microenvironment on mitochondrial dynamics and chemoresistance in cancer. Free Radic Res 2018:1-17.

[52] DiGiacomo JW, Gilkes DM. Tumor hypoxia as an enhancer of inflammation-mediated metastasis: emerging therapeutic strategies. Target Oncol 2018;13(2):157-73.

[53] Pierobon D, Bosco MC, Blengio F, Raggi F, Eva A, Filippi M, et al. Chronic hypoxia reprograms human immature dendritic cells by inducing a proinflammatory phenotype and TREM-1 expression. Eur J Immunol 2013;43(4):949-66.

[54] Bartels K, Grenz A, Eltzschig HK. Hypoxia and inflammation are two sides of the same coin. Proc Natl Acad Sci U S A 2013; 110(46):18351-2.

[55] Fawzy MS, Toraih EA, Ibrahiem A, Abdeldayem H, Mohamed AO, Abdel-Daim MM. Evaluation of miRNA-196a2 and apoptosis-related target genes: ANXA1, DFFA and PDCD4 expression in gastrointestinal cancer patients: a pilot study. PLoS One 2017;12(11):e0187310.

[56] Luthra R, Singh RR, Luthra MG, Li YX, Hannah C, Romans AM, et al. MicroRNA-196a targets annexin A1: a microRNA-mediated mechanism of annexin A1 downregulation in cancers. Oncogene 2008;27(52):6667-78.

[57] Nomura H, Uzawa K, Yamano Y, Fushimi K, Nakashima D, Kouzu Y, et al. Down-regulation of plasma membranous Annexin A1 protein expression in premalignant and malignant lesions of the oral cavity: correlation with epithelial differentiation. J Cancer Res Clin Oncol 2009;135(7):943-9.

[58] Alves VA, Nonogaki S, Cury PM, Wünsch-Filho V, De Carvalho MB, Michaluart-Júnior P, et al. Annexin A1 subcellular expression in laryngeal squamous cell carcinoma. Histopathology 2008:53(6):715-27.
[59] Yi M, Schnitzer JE. Impaired tumor growth, metastasis, angiogenesis and wound healing in annexin A1-null mice. Proc Natl Acad Sci U S A 2009;106(42):17886-91.

[60] Gastardelo TS, Cunha BR, Raposo LS, Maniglia JV, Cury PM, Lisoni FC, et al. Inflammation and cancer: role of annexin A1 and FPR2/ALX in proliferation and metastasis in human laryngeal squamous cell carcinoma. PLoS One 2014;9(12):e111317.

[61] Maschler S, Gebeshuber CA, Wiedemann EM, Alacakaptan M, Schreiber M, Custic I, et al. Annexin A1 attenuates EMT and metastatic potential in breast cancer. EMBO Mol Med 2010; 2(10):401-14.

[62] Bizzarro V, Belvedere R, Milone MR, Pucci B, Lombardi R, Bruzzese F, et al. Annexin A1 is involved in the acquisition and maintenance of a stem cell-like/aggressive phenotype in prostate cancer cells with acquired resistance to zoledronic acid. Oncotarget 2015;6(28):25076-92.

[63] Zhang F, Wang Z, Yuan J, Wei X, Tian R, Niu R. RNAimediated silencing of Anxa2 inhibits breast cancer cell proliferation by downregulating cyclin D1 in STAT3-dependent pathway. Breast Cancer Res Treat 2015;153(2):263-75.

[64] Shetty P, Bargale A, Patil BR, Mohan R, Dinesh US, Vishwanatha JK, et al. Cell surface interaction of annexin A2 and galectin-3 modulates epidermal growth factor receptor signaling in Her-2 negative breast cancer cells. Mol Cell Biochem 2016; 411(1-2):221-33.

[65] Ries J, Vairaktaris E, Agaimy A, Bechtold M, Gorecki P, Neukam FW, et al. The relevance of EGFR overexpression for the prediction of the malignant transformation of oral leukoplakia. Oncol Rep 2013;30(3):1149-56.

[66] Patel V, Ramesh A, Traicoff JL, Baibakov G, Emmert-Buck MR, Gutkind JS, et al. Profiling EGFR activity in head and neck squamous cell carcinoma by using a novel layered membrane Western blot technology. Oral Oncol 2005;41(5):503-8.

[67] Liao JB, Lee HP, Fu HT, Lee HS. Assessment of EGFR and ERBB2 (HER2) in gastric and gastroesophageal carcinomas: EGFR amplification is associated with a worse prognosis in early stage and well to moderately differentiated carcinoma. Appl Immunohistochem Mol Morphol 2018 Jul;26(6):374-82.

[68] Chen HY, Liu CH, Chang YH, Yu SL, Ho BC, Hsu CP, et al. EGFR-activating mutations, DNA copy number abundance of ErbB family, and prognosis in lung adenocarcinoma. Oncotarget 2016;7(8):9017-25.

[69] Jacot W, Mollevi C, Fina F, Lopez-Crapez E, Martin PM, Colombo PE, et al. High EGFR protein expression and exon 9 PIK3CA mutations are independent prognostic factors in triple negative breast cancers. BMC Cancer 2015;15:986.

[70] Hiraishi Y, Wada T, Nakatani K, Negoro K, Fujita S. Immunohistochemical expression of EGFR and p-EGFR in oral squamous cell carcinomas. Pathol Oncol Res 2006;12(2):87-91.

[71] Gao J, Ulekleiv CH, Halstensen TS. Epidermal growth factor (EGF) receptor-ligand based molecular staging predicts prognosis in head and neck squamous cell carcinoma partly due to deregulated EGF- induced amphiregulin expression. J Exp Clin Cancer Res 2016;35(1):151.

[72] Chen YJ, Chen SY, Lovel R, Ku YC, Lai YH, Hung CL, et al. Enhancing chemosensitivity in oral squamous cell carcinoma by lentivirus vector-mediated RNA interference targeting EGFR and MRP2. Oncol Lett 2016;12(3):2107-14.

[73] Nozawa H, Tadakuma T, Ono T, Sato M, Hiroi S, Masumoto K, et al. Small interfering RNA targeting epidermal growth factor receptor enhances chemosensitivity to cisplatin, 5-fluorouracil and docetaxel in head and neck squamous cell carcinoma. Cancer Sci 2006;97(10):1115-24.

[74] van Dommelen SM, van der Meel R, van Solinge WW, Coimbra M, Vader P, Schiffelers RM. Cetuximab treatment alters the content of extracellular vesicles released from tumor cells. Nanomedicine (Lond) 2016;11(8):881-90. 
[75] Jones S, Rappoport JZ. Interdependent epidermal growth factor receptor signalling and trafficking. Int J Biochem Cell Biol 2014; $51: 23-8$.

[76] Monteiro LS, Ricardo S, Delgado ML, Garcez F, do Amaral B, Lopes C. Phosphorylated EGFR at tyrosine 1173 correlates with poor prognosis in oral squamous cell carcinomas. Oral Dis 2014; 20(2):178-85.

[77] Aquino G, Pannone G, Santoro A, Liguori G, Franco R, Serpico R, et al. pEGFR-Tyr 845 expression as prognostic factors in oral squamous cell carcinoma: a tissue-microarray study with clinic-pathological correlations. Cancer Biol Ther 2012;13(11): 967-77.
[78] Schneider T, Strehl A, Linz C, Brands R, Hartmann S, Beckford F, et al. Phosphorylated epidermal growth factor receptor expression and KRAS mutation status in salivary gland carcinomas. Clin Oral Investig 2016;20(3):541-51.

[79] Hessvik NP, Llorente A. Current knowledge on exosome biogenesis and release. Cell Mol Life Sci 2018 Jan;75(2):193-208.

[80] Matsumoto Y, Kano M, Akutsu Y, Hanari N, Hoshino I, Murakami K, et al. Quantification of plasma exosome is a potential prognostic marker for esophageal squamous cell carcinoma. Oncol Rep 2016;36(5):2535-43.

[81] Dvorak HF. Tumors: wounds that do not heal-redux. Cancer Immunol Res 2015;3(1):1-11. 